\title{
Pneumothorax as a Presenting Clinical Manifestation of Metastatic Prostate Cancer
}

\author{
Kuo-Hwa Chiang ${ }^{\mathrm{a}, \mathrm{b}, \mathrm{e}}$, Shun-Hsing Hung ${ }^{\mathrm{c}}$, Sheng-Tsung Chang ${ }^{\mathrm{d}}$
}

\begin{abstract}
Pulmonary metastases are not encountered commonly in patients with prostate cancer. Pulmonary metastases with pneumothorax as a presenting clinical manifestation in newly diagnosed prostate cancer are very rare. Here, we present the case of an 82-year-old patient who was admitted to our center with a chief complaint of worsening dyspnea over the past few days. The chest X-ray and computed tomography (CT) showed left pneumothorax and bilateral lung opacities as well as generalized lymphadenopathy and diffuse bony metastases. After a series of workup including cervical lymph node biopsy with immunohistochemical staining, abdomen CT, serum prostate-specific antigen (PSA), and transrectal ultrasonography (TRUS), he was proved to have prostate cancer with multiple lung, bone and lymph node metastases. This case is reported because of the rarity for a prostate carcinoma presented clinically with an unusual pulmonary manifestation.
\end{abstract}

Keywords: Prostate adenocarcinoma; Pneumothorax; Cervical lymph node; Pulmonary metastases

\section{Introduction}

Prostate cancer is the most common noncutaneous human malignancy, with an estimated 241,740 new cases in the USA in 2012. It is also the second-most lethal tumor among men, with 28,170 expected deaths in 2012 [1]. The most common sites of metastases of prostate are regional lymph nodes and

\footnotetext{
Manuscript accepted for publication January 28, 2013

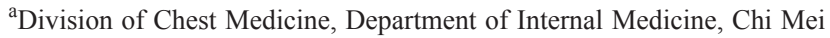
Medical Center, Tainan, Taiwan

${ }^{\mathrm{b}}$ Department of Information Management, Chia Nan University of Pharmacy and Science, Tainan, Taiwan

${ }^{\mathrm{c}}$ Department of Urology, Chi Mei Medical Center, Tainan, Taiwan

${ }^{\mathrm{d}}$ Department of Pathology, Chi Mei Medical Center, Tainan, Taiwan

${ }^{\mathrm{e}}$ Corresponding author: Kuo-Hwa Chiang, No.901, Chun-Hua Rd. Yong Kang Dist, Tainan City 710, Taiwan (ROC).

Email: chiangkh2003@yahoo.com.tw
}

doi: http://dx.doi.org/10.4021/wjon630w bones, followed by lung, bladder, liver, adrenal gland and kidney. Prostate cancer with supraclavicular lymph nodes metastases is rare, with a reported incidence of approximately $0.28 \%$ [2]. Pulmonary metastases with pneumothorax as a presenting clinical manifestation in newly diagnosed prostate cancer are very rare. Here, we present an unusual case of 82 -year-old male without previous cancer history, who admitted to our hospital due to pneumothorax with collapse of left lung, and after a series of workup he was proved to have prostate cancer with lung and left lower cervical, left supraclavicular, mediastinum and abdomen lymph node and bony metastases. To our knowledge, this is the first case report in English literature.

\section{Case Report}

An 82-year-old male patient presented with chest tightness and shortness of breathing for 10 days. His past medical history included hypertension and old pulmonary tuberculosis. He suffered from productive cough for weeks and was referred to our center because of the worsening symptoms of dyspnea and lower leg edema noted over the past few days. On presentation, his consciousness was alert. Body temperature was $37.8{ }^{\circ} \mathrm{C}$. Pulse was 136 per minute regular. Blood pressure was $164 / 90 \mathrm{mmHg}$. Oxygen saturation was $88 \%$. Auscultation revealed decreased breathing sounds at left lung field and sonorous rhonchus at right lung field. X-ray of the chest showed left pneumothorax with collapse of left lung, and ground glass opacities in right lung field (Fig. 1), more in favor of inflammatory process at that time. He was admitted under the impression of right lung pneumonia with left pneumothorax and received chest tube insertion and empiric antibiotics treatment. However persistent air leak noted, he then received a chest $\mathrm{CT}$ for further survey. A chest CT showed nodular lesions over bilateral lung, with diffuse bone, left lower neck (Fig. 2), left suparclavicular, mediastinum, as well as right hilar (Fig. 3) and lower abdominal lymph nodes metastases. Unknown primary malignancy with bilateral lung metastases, diffuse bony metastases with left supraclavicular and left neck lymph node metastases was impressed and further tissue proof for possible under- 


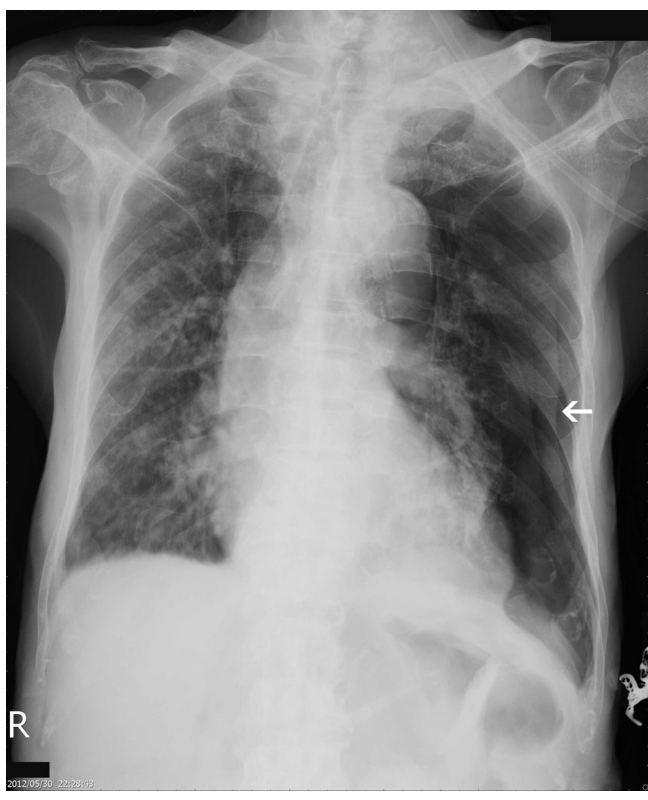

Figure 1. Initial CXR showing left pneumothorax, with ground grass densities over right lung.

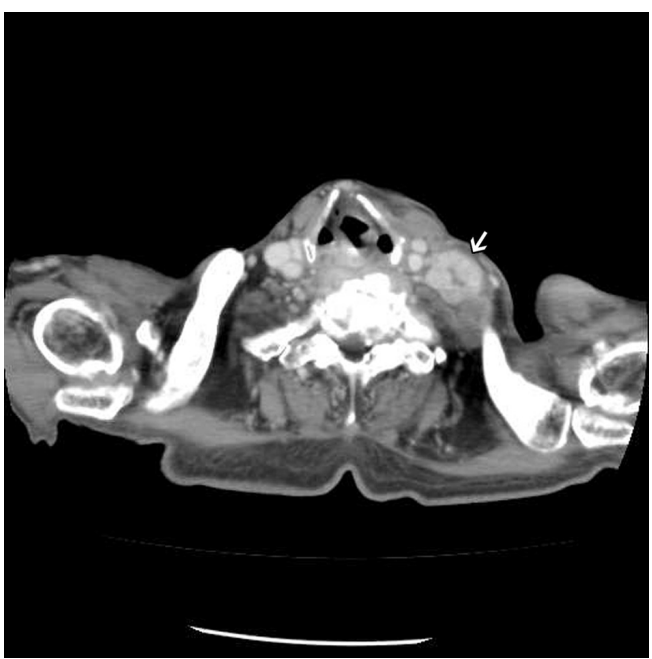

Figure 2. Enhanced chest CT showing an enlarged lymph node at left lower neck.

lying malignancy was recommended. For this reason, an echo guided biopsy with left neck lymph node biopsy was arranged for further study because the surgical intervention was not recommended for the patient due to poor lung function while patient declined bronchoscopy biopsy either. A subsequent neck lymph node biopsy revealed metastatic adenocarcinoma, while immunohistochemical stain for thyroid transcription factor -1(TTF-1), and cytokeratin 20 (CK20) are all negative. Serum tumor markers were examined in an attempt to identify the primary lesion, revealing a PSA level of $>1,000 \mathrm{ng} / \mathrm{mL}$ (normal $<4.0 \mathrm{ng} / \mathrm{mL}$ ) while CEA $2.7 \mathrm{ng} /$

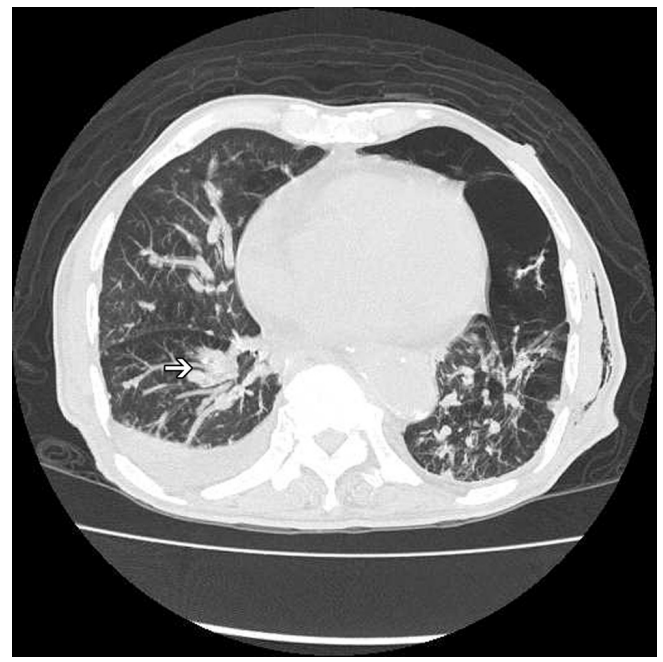

Figure 3. High resolution computed tomography (HRCT) of the chest showing hilar lymphadenopathy (arrow) and diffuse reticular nodular lesions over bilateral lung, with left residule pneumothorax and subcutaneous emphysema over left chest wall.

$\mathrm{mL}$ (normal $<5.0 \mathrm{ng} / \mathrm{mL}$ ). Digital rectal examination showed an enlarged and hardened left prostate lobe. An abdomen CT disclosed $4.3 \times 3.0 \mathrm{~cm}$ ill defined heterogenous mass lesion at lower lobe of prostate (Fig. 4a) with regional and nonregional lymph nodes (Fig. 4b) and bony metastases. The urologist was consulted, and transrectal ultrasonography (TRUS) showed irregular urinary bladder wall thickening with enlargement of prostate, hypoechoic nodule with increased vascularity in left peripheral zone of prostate. Meanwhile the patient refused further tissue proof due to his poor general condition. The pathologist was informed of the elevated serum PSA and further clinical data, then an additional immunohistochemical staining of the left neck lymph node biopsy with P504S was performed as was compared to the regular H\&E stain (Fig. 5a), the neoplastic cells expressed P504S diffusely (Fig. 5b), a metastatic adenocarcinoma of prostatic origin was confirmed. However his condition went downhill rapidly, and after thoroughly discussion with the patient and his family, they decided to receive hospice care and refused further intervention.

\section{Discussion}

This case is unique because it presents pneumothorax as the initial manifestation of prostate cancer with generalized lymphadenopathy and lung metastases. This atypical presentation has made diagnosing prostate adenocarcinoma extremely difficult.

Prostate cancer metastases to mediastinal, supraclavicular, and cervical lymph nodes are uncommon [3]. The incidence of cervical lymph node involvement in patients with 


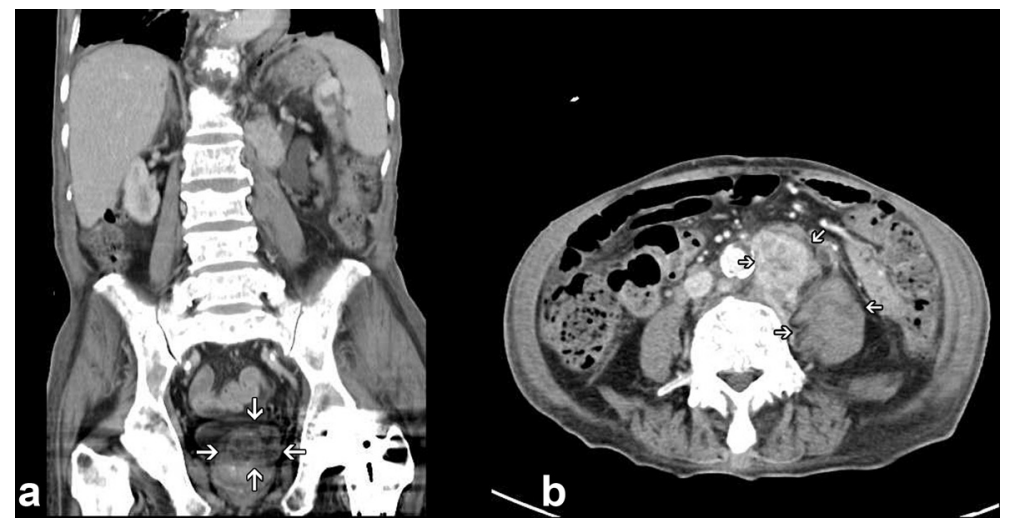

Figure 4. Enhanced lower abdomen CT showing a $4.3 \times 3.0 \mathrm{~cm}$ ill defined heterogenous mass lesion over left lobe of prostate (a); with extensive para-aortic and retroperitoneal lymphadenopathy (b).

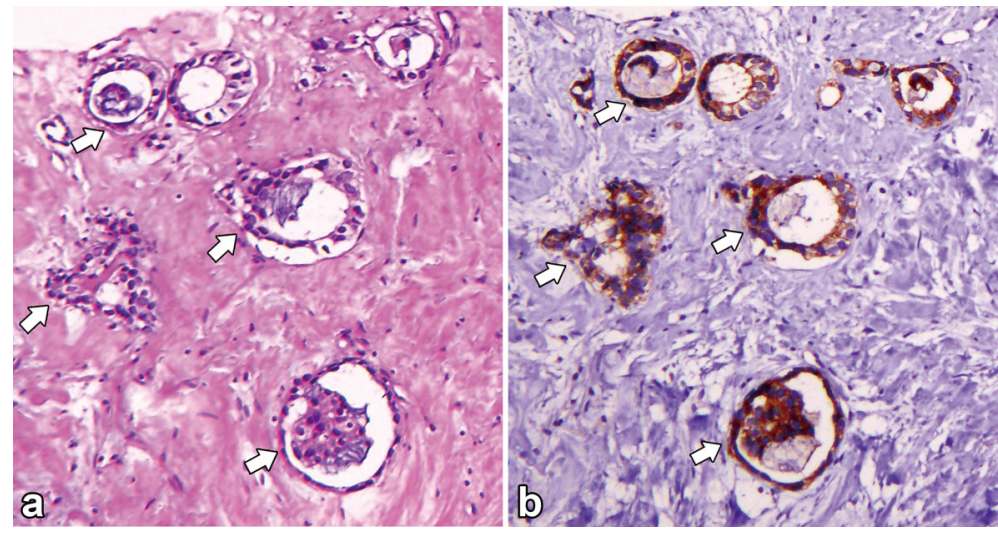

Figure 5. (a) Hematoxyline and Eosin (H\&E) stain of biopsy specimen of the left lower neck lymph node showing tubuloglandular structures with stromal fibrosis; (b) Immunohistochemical stain with P504s showing neoplastic cells express P504s diffusely.

prostate cancer has been reported as $0.28 \%$; however, these patients almost uniformly present with widespread metastatic disease [4-7]. In this case, the history and the symptoms signs the patient presented didn't offer much that would help guide the clinician towards the correct primary site of disease. His initial presentations of pneumothorax along with left neck lymphadenopathy once lead our attention to primary lung malignancy. However, after his cervical lymph node biopsy revealed metastatic adenocarcinoma, with a negative TTF1, further series of workup for unknown primary including abdominal CT, transrectal ultrasonography (TRUS)and serum PSA as well as special immunohistochemistry stain of the pathology specimen with P504S had performed. P504S is the first gene identified from prostate cancer by cDNA microarrays to be suitable for clinical practice and to potentially improve the diagnosis of prostate cancer [8]. In distinction to PSA, P504S is highly selective for prostate cancer. A number of studies from several institutions have demonstrated that P504S is an important positive tissue marker for prostate carcinoma regardless of tumor grade $[8,9]$. In this case, imaging studies, immunohistochemical stains, and pathological specimens all pointed to a narrow differential, serum tumor markers were obtained to further help in the diagnosis of metastatic prostate cancer. Pulmonary metastases are common in advanced prostate carcinoma, with autopsy studies showing an incidence of $25 \%$ to $50 \%$ [10] but are rare at diagnosis. Pulmonary manifestations are clinically recognized in only $5 \%$ of cases. Metastatic prostate cancer with pneumothorax is extremely rare. The exact mechanism is not clear, but it might be possibly related to extensive lung metastases from prostate cancer. Pulmonary metastases are seen concomitant with lymph node and bone metastases and are most commonly encountered with multi-organ involvement $[10,11]$. Multiple spread of carcinoma to the lung is a form of the terminal phase of carcinoma and leads to a poor prognosis. It may have progressed considerably when multiple lesions were observed in lungs, and may follow a miserable course as described in the present case. 
The case is an aggressive prostate cancer with a very unusual presentation. Although metastases to the cervical lymph nodes are rare in prostate cancer patients, they should be considered as one of the differential diagnoses of cervical lymphadenopathy in elderly males with adenocarcinoma of undetermined origin even in an atypical presentation. Serum PSA level and subsequent immunohistochemical stains of their lymph node biopsies can be helpful in establishing a definitive diagnosis of prostate cancer.

\section{Grant Support}

None.

\section{Conflict of Interests}

We declare that the content has not been previously published and has been submitted solely to World Journal of Oncology. We declare no conflict of interest. This article did not receive any funding from any institution.

\section{References}

1. Siegel R, Naishadham D, Jemal A. Cancer statistics, 2012. CA Cancer J Clin. 2012;62(1):10-29.

2. Hematpour K, Bennett CJ, Rogers D, Head CS. Supraclavicular lymph node: incidence of unsuspected metastatic prostate cancer. Eur Arch Otorhinolaryngol. 2006;263(9):872-874.
3. Cho KR, Epstein JI. Metastatic prostatic carcinoma to supradiaphragmatic lymph nodes. A clinicopathologic and immunohistochemical study. Am J Surg Pathol. 1987;11(6):457-463.

4. Saitoh H, Yoshida K, Uchijima Y, Kobayashi N, Suwata J, Kamata S. Two different lymph node metastatic patterns of a prostatic cancer. Cancer. 1990;65(8):18431846.

5. Copeland B, Clark JM, Sura A, Kilpatrick SE, Shockley W, Meredith S. Prostate carcinoma metastatic to the cervical lymph nodes: report of two cases and review of the literature. Am J Otolaryngol. 2001;22(6):420-423.

6. Carleton J, van der Riet P, Dahm P. Metastatic prostate cancer presenting as an asymptomatic neck mass. Prostate Cancer Prostatic Dis. 2005;8(3):293-295; discussion 295.

7. Oyan B, Engin H, Yalcin S. Generalized lymphadenopathy: a rare presentation of disseminated prostate cancer. Med Oncol. 2002;19(3):177-179.

8. Jiang Z, Woda BA, Wu CL, Yang XJ. Discovery and clinical application of a novel prostate cancer marker: alpha-methylacyl CoA racemase (P504S). Am J Clin Pathol. 2004;122(2):275-289.

9. Hameed O, Humphrey PA. Immunohistochemistry in diagnostic surgical pathology of the prostate. Semin Diagn Pathol. 2005;22(1):88-104.

10. Mestitz H, Pierce RJ, Holmes PW. Intrathoracic manifestations of disseminated prostatic adenocarcinoma. Respir Med. 1989;83(2):161-166.

11. Fabozzi SJ, Schellhammer PF, el-Mahdi AM. Pulmonary metastases from prostate cancer. Cancer. 1995;75(11):2706-2709. 